\title{
ON TRANS-SASAKIAN MANIFOLDS
}

\author{
Riddhi Jung Shah \\ Department of Mathematics \& Astronomy \\ University of Lucknow, Lucknow-226007 (India) \\ Corresponding address: shahrjgeo@gmail.com \\ Received 02 January, 2011; Revised 29 December, 2011
}

\begin{abstract}
In this paper we study the geometry of trans-Sasakian manifold when it is projective Ricci-semi-symmetric, pseudo-projectively flat and pseudo-projectively semi-symmetric.
\end{abstract}

MSC 2010: 53C15, 53C25, 55S10.

Keywords: Trans-Sasakian manifold, projective Ricci tensor, pseudo-projective curvature tensor, pseudo-projectively flat, pseudo-projectively semi-symmetric.

\section{INTRODUCTION}

In 1985, J. A. Oubina [9] introduced the notion of trans-Sasakian manifold. Many geometers studied this manifold some of them are [9, 7, 1]. Semi-symmetric manifold is studied by author [10], [11] and others. The conditions $R(X, Y) \cdot \tilde{P}=0, \quad \bar{P}(X, Y) Z=0$ and $R(X, Y) \cdot \bar{P}=0$ are called projective Ricci-semi-symmetric, pseudo-projectively flat and pseudo-projectively semisymmetric respectively, where $R(X, Y)$ is considered as derivation of tensor algebra at each point of the manifold.

We note that trans-Sasakian structure of type $(0,0),(0, \beta)$ and $(\alpha, 0)$ are called cosympletic, $\beta$-Kenmotsu and $\alpha$-Sasakian manifold respectively. Thus trans-Sasakian structures are also provide a large class of generalized quasi-Sasakian structures.

An almost contact metric manifold $M^{2 n+1}(\phi, \xi, \eta, g)$ is said to be trans-Sasakian manifold [1] if $(M \times \square, J, G)$ belongs to the class $\omega_{4}[8]$ of the Hermitian manifolds where $\mathrm{J}$ is the almost complex structure on $M \times \square$ defined by

$$
J\left(X, f \frac{d}{d t}\right)=\left(\phi X-f \xi, \eta(X) f \frac{d}{d t}\right),
$$

for all vector fields on $\mathrm{M}$ and smooth function $\mathrm{f}$ on $M \times \square$ and $\mathrm{G}$ is the product metric on $M \times \square$. This may be stated by the condition [4]

$$
\left(\nabla_{X} \phi\right)(Y)=\alpha\{g(X, Y) \xi-\eta(Y) X\}+\beta\{g(\phi X, Y) \xi-\eta(Y) \phi X\},
$$

for some smooth functions $\alpha$ and $\beta$ on $\mathrm{M}$ and we say that trans-Sasakian structure is of type $(\alpha, \beta)$.

In this paper we consider the trans-Sasakian manifold under the condition $\phi(\operatorname{grad} \alpha)=(2 n-1) \operatorname{grad} \beta \quad$ satisfying $\quad R(X, Y) . \tilde{P}=0, \quad \bar{P}(X, Y) Z=0 \quad$ and $R(X, Y) \cdot \bar{P}=0$, where $\tilde{P}$ is the projective Ricci tensor introduced by the authors [6]. It is defined by 


$$
\tilde{P}(X, Y)=\frac{(2 n+1)}{2 n} S(X, Y)-\frac{r}{2 n} g(X, Y),
$$

where $S$ and $r$ are Ricci tensor and scalar curvature respectively. It is shown that in first condition the manifold is Einstein and its scalar curvature is $2 n(2 n+1)\left(\alpha^{2}-\beta^{2}\right)$.

Further, trans-Sasakian manifold $M^{2 n+1}(\phi, \xi, \eta, g)$ with $\bar{P}(X, Y) Z=0$ and $R(X, Y) . \bar{P}=0$, is considered, where $\bar{P}$ is the pseudo-projective curvature tensor given by [2]

$$
\begin{aligned}
\bar{P}(X, Y) Z & =a R(X, Y) Z+b[S(Y, Z) X-S(X, Z) Y] \\
& -\frac{r}{(2 n+1)}\left\{\frac{a}{2 n}+b\right\}[g(Y, Z) X-g(X, Z) Y],
\end{aligned}
$$

where $a, b$ are constants such that $a, b \neq 0, R, S, r$ are the curvature tensor, Ricci tensor and scalar curvature respectively.

\section{PRELIMINARIES}

Let $M$ be a $(2 n+1)$-dimensional almost contact metric manifold [3] with an almost contact metric structure $(\phi, \xi, \eta, g)$, where $\phi$ is a $(1,1)$ tensor field, $\xi$ is a vector field, $\eta$ is a 1 -form and $\mathrm{g}$ is the associated Riemannian metric such that

$$
\begin{gathered}
\phi^{2}(X)=-X+\eta(X) \xi, \\
\eta(\xi)=g(\xi, \xi)=1, \phi \xi=0, \\
\eta(\phi X)=0, \eta \circ \phi=0, \\
g(\phi X, \phi Y)=g(X, Y)-\eta(X) \eta(Y), g(X, \xi)=\eta(X), \\
d \eta(X, Y)=g(X, \phi Y)=-g(\phi X, Y), \text { for all } X, Y \in T M .
\end{gathered}
$$

From (1.2) it follows that

$$
\begin{gathered}
\nabla_{X} \xi=-\alpha \phi X+\beta\{X-\eta(X) \xi\}, \\
\left(\nabla_{X} \eta\right)(Y)=-\alpha g(\phi X, Y)+\beta g(\phi X, \phi Y) .
\end{gathered}
$$

Further, on a trans-Sasakian manifold the following relations hold [7], [5]:

$$
\begin{gathered}
R(X, Y) \xi=\left(\alpha^{2}-\beta^{2}\right)[\eta(Y) X-\eta(X) Y]-(X \alpha) \phi Y-(X \beta) \phi^{2} Y \\
+2 \alpha \beta[\eta(Y) \phi X-\eta(X) \phi Y]+(Y \alpha) \phi X+(Y \beta) \phi^{2} X, \\
R(\xi, X) \xi=\left(\alpha^{2}-\beta^{2}-\xi \beta\right)[\eta(X) \xi-X], \\
2 \alpha \beta+(\xi \alpha)=0, \\
S(X, \xi)=\left[2 n\left(\alpha^{2}-\beta^{2}\right)-(\xi \beta)\right] \eta(X)-(\phi X) \alpha-(2 n-1)(X \beta), \\
Q \xi=\left[2 n\left(\alpha^{2}-\beta^{2}\right)-(\xi \beta)\right] \xi+\phi(\operatorname{grad} \alpha)-(2 n-1) \operatorname{grad} \beta,
\end{gathered}
$$

when $\phi(\operatorname{grad} \alpha)=(2 n-1) \operatorname{grad} \beta$, then the relations (2.11) and (2.12) reduce to 


$$
\begin{gathered}
S(X, \xi)=2 n\left(\alpha^{2}-\beta^{2}\right) \eta(X), \\
Q \xi=2 n\left(\alpha^{2}-\beta^{2}\right) \xi, \\
S(\xi, \xi)=2 n\left(\alpha^{2}-\beta^{2}\right) .
\end{gathered}
$$

\section{RESULTS AND DISCUSSION}

Theorem 3.1: If in a trans-Sasakian manifold $M^{2 n+1}(\phi, \xi, \eta, g)$, the relation $R(X, Y) . \tilde{P}=0$ holds, then the manifold is Einstein.

Proof: Consider a trans-Sasakian manifold $M^{2 n+1}(\phi, \xi, \eta, g)$ which satisfies the condition

$$
R(X, Y) \cdot \tilde{P}=0
$$

where $\tilde{P}$ is the projective Ricci Tensor defined in (1.3). Now,

$$
(R(X, Y) . \tilde{P})(U, V)=-\tilde{P}(R(X, Y) U, V)-\tilde{P}(U, R(X, Y) V) .
$$

From (3.1) and (3.2), we get

$$
\tilde{P}(R(X, Y) U, V)+\tilde{P}(U, R(X, Y) V)=0 .
$$

Putting $X=\xi$ and using (2.8) in (3.3) we have

$$
\begin{aligned}
& \left(\alpha^{2}-\beta^{2}\right)[g(Y, U) \tilde{P}(\xi, V)-\eta(U) \tilde{P}(Y, U)+g(Y, U) \tilde{P}(\xi, U)-\eta(V) \tilde{P}(U, Y)] \\
& -2 \alpha \beta[\eta(U) \tilde{P}(\phi Y, V)+\eta(V) \tilde{P}(\phi Y, U)]-(\xi \alpha)[\tilde{P}(\phi Y, V)+\tilde{P}(\phi Y, U)]=0
\end{aligned}
$$

Putting $V=U$ in (3.4), we get

$$
\left(\alpha^{2}-\beta^{2}\right)[g(Y, U) \tilde{P}(\xi, U)-\eta(U) \tilde{P}(Y, U)]-\tilde{P}(\phi Y, U)[2 \alpha \beta \eta(U)+(\xi \alpha)]=0 .
$$

Under condition $2 \alpha \beta \eta(U)+\xi \alpha=0$ if $\eta(U)=1$, using (3) and (2.13) in (3.5), we get

$$
S(U, Y)=2 n\left(\alpha^{2}-\beta^{2}\right) g(U, Y) \text {. }
$$

This implies that the manifold is an Einstein manifold. This completes the proof of the theorem.

Let $\left\{e_{i}: i=1,2, \ldots, 2 n+1\right\}$ be an orthonormal basis of the tangent space at any point of the manifold. Putting $U=Y=e_{i}$ in (3.6) and taking summation over i, $1 \leq i \leq 2 n+1$, we get

$$
r=2 n(2 n+1)\left(\alpha^{2}-\beta^{2}\right) \text {. }
$$

Hence we can state:

Corollary 3.1: A projective Ricci-semi-symmetric trans-Sasakian manfold $M^{2 n+1}(\phi, \xi, \eta, g)$, is the manifold of constant scalar curvature $2 n(2 n+1)\left(\alpha^{2}-\beta^{2}\right)$.

Theorem 3.2: A pseudo-projectively flat trans-Sasakian manfold $M^{2 n+1}(\phi, \xi, \eta, g)$ is an $\eta$-Einsten manifold provided that $a, b \neq 0$.

Proof: The pseudo-projective curvature tensor is given by the relation (4). Suppose $\bar{P}(X, Y) Z=0$, then from (1.4), we get

$$
\begin{gathered}
a R(X, Y) Z+b[S(Y, Z) X-S(X, Z) Y] \\
-\frac{r}{(2 n+1)}\left\{\frac{a}{2 n}+b\right\}[g(Y, Z) X-g(X, Z) Y]=0
\end{gathered}
$$


Taking inner product on both sides of (3.2.1) by $\xi$, we get

$$
\begin{aligned}
& a \eta(R(X, Y) Z)+b[S(Y, Z) \eta(X)-S(X, Z) \eta(Y)] \\
& -\frac{r(a+2 n b)}{2 n(2 n+1)}[g(Y, Z) \eta(X)-g(X, Z) \eta(Y)]=0
\end{aligned}
$$

Putting $X=\xi$ and using (2.4),(2.8) and (2.13), in (3.2.2), we get

$$
\begin{gathered}
a\left[\left(\alpha^{2}-\beta^{2}\right)\{g(Y, Z)-\eta(Y) \eta(Z)\}\right]+b\left[S(Y, Z)-2 n\left(\alpha^{2}-\beta^{2}\right) \eta(Y) \eta(Z)\right] \\
-\left\{\frac{r(a+2 n b)}{2 n(2 n+1)}\right\}[g(Y, Z)-\eta(Y) \eta(Z)]=0
\end{gathered}
$$

which yields on further calculation

$$
\begin{aligned}
& S(Y, Z)=\left[\frac{1}{b}\left\{\frac{(a+2 n b) r}{2 n(2 n+1)}-a\left(\alpha^{2}-\beta^{2}\right)\right\}\right] g(Y, Z) \\
& +\left[\frac{(a+2 n b)}{b}\left\{\left(\alpha^{2}-\beta^{2}\right)-\frac{r}{2 n(2 n+1)}\right\}\right] \eta(Y) \eta(Z) .
\end{aligned}
$$

Thus the theorem is proved.

Let $\left\{e_{i}: i=1,2, \ldots ., 2 n+1\right\}$ be an orthonormal basis of the tangent space at any point of the manifold. Putting $Y=Z=e_{i}$ in (3.2.3) and taking summation over i, $1 \leq i \leq 2 n+1$, we get

$$
r=2 n(2 n+1)\left(\alpha^{2}-\beta^{2}\right) \text {. }
$$

Hence we can state:

Corollary 3.2: A pseudo-projectively flat trans-Sasakian manifold of dimension $(2 n+1)$ is of manifold of constant scalar curvature $2 n(2 n+1)\left(\alpha^{2}-\beta^{2}\right)$.

Using the relation (3.2.4) in (3.2.3), we get

$$
S(Y, Z)=2 n\left(\alpha^{2}-\beta^{2}\right) g(Y, Z) \text {. }
$$

This leads to the following:

Theorem 3.3: If a trans-Sasakian manifold $M^{2 n+1}(\phi, \xi, \eta, g)$ is pseudo-projectively flat then it is Einstein one \& its scalar curvature is given by (3.2.4).

Theorem 3.4: A pseudo-projectively semi-symmetric trans-Sasakian manifold $M^{2 n+1}(\phi, \xi, \eta, g)$ is an $\eta$-Einstein manifold.

Proof: Let us suppose that a $(2 n+1)$-dimensional trans-Sasakian manifold satisfies the condition

$$
\mathrm{R}(\mathrm{X}, \mathrm{Y}) \cdot \overline{\mathrm{P}}=0
$$

where $\overline{\mathrm{P}}$ is the pseudo-projective curvature tensor given in (1.4).

Using (2.4) and (2.8) in (1.4), we get 


$$
\begin{aligned}
\eta(\bar{P}(U, V) W) & =\left\{a\left(\alpha^{2}-\beta^{2}\right)-\frac{r(a+2 n b)}{2 n(2 n+1)}\right\}[g(U, V) \eta(U)-g(U, W) \eta(V)] \\
& +b[S(V, W) \eta(U)-S(U, W) \eta(V)] .
\end{aligned}
$$

Taking $U=\xi$ in (3.4.2) and using (2.2), (2.4) and (2.13), we get

$$
\begin{aligned}
\eta(\bar{P}(\xi, V) W)= & b S(V, W)\left\{a\left(\alpha^{2}-\beta^{2}\right)-\frac{(a+2 n b) r}{2 n(2 n+1)}\right\} g(V, W) \\
& +\left[(a+2 n b)\left\{\frac{r}{2 n(2 n+1)}-\left(\alpha^{2}-\beta^{2}\right)\right\}\right] \eta(V) \eta(W) .
\end{aligned}
$$

Putting $W=\xi$ in (3.4.2) and using (2.8) and (2.13), we obtain

$$
\eta(\bar{P}(U, V) \xi)=0 \text {. }
$$

Now,

$$
\begin{aligned}
(R(X, Y) . \bar{P})(U, V) W & =R(X, Y) \bar{P}(U, V) W-\bar{P}(R(X, Y) U, V) W \\
& -\bar{P}(U, R(X, Y) V) W-\bar{P}(U, V) R(X, Y) W .
\end{aligned}
$$

From the relations (5.1) and (5.5), we have

$$
\begin{gathered}
R(X, Y) \bar{P}(U, V) W-\bar{P}(R(X, Y) U, V) W-\bar{P}(U, R(X, Y) V) W \\
-\bar{P}(U, V) R(X, Y) W=0 .
\end{gathered}
$$

Putting $X=\xi$ and taking inner product on both sides of (3.4.6) by $\xi$, we get

$$
\begin{gathered}
\eta(R(\xi, Y) \bar{P}(U, V) W)-\eta(\bar{P}(R(\xi, Y) U, V) W)-\eta(\bar{P}(U, R(\xi, Y) V) W) \\
-\eta(\bar{P}(U, V) R(\xi, Y) W)=0 .
\end{gathered}
$$

From this it follows that

$$
\begin{gathered}
\bar{P}(U, V, W, Y)-\eta(Y) \eta(\bar{P}(U, V) W)-g(Y, U) \eta(\bar{P}(\xi, V) W)+\eta(U) \eta(\bar{P}(Y, V) W) \\
+\eta(W) \eta(\bar{P}(U, V) Y)-g(Y, V) \eta(\bar{P}(U, \xi) W)+\eta(V) \eta(\bar{P}(U, Y) W)=0,
\end{gathered}
$$

where $\bar{P}(U, V, W, Y)=g(\bar{P}(U, V) W, Y)$.

Putting $Y=U$ in (3.4.8), we get

$$
\begin{gathered}
\bar{P}(U, V, W, U)-g(U, U) \eta(\bar{P}(\xi, V) W)-g(U, V) \eta(\bar{P}(U, \xi) W) \\
+\eta(V) \eta(\bar{P}(U, U) W)+\eta(W) \eta(\bar{P}(U, V) U)=0 .
\end{gathered}
$$

Let $\left\{e_{i}: i=1,2, \ldots, 2 n+1\right\}$ be an orthonormal basis of the tangent space at any point of the manifold. Then the sum for $1 \leq i \leq 2 n+1$ of the relation (3.4.9) for $U=e_{i}$, yields

$$
\begin{aligned}
\eta(\bar{P}(\xi, V) W) & =\left(\frac{a+2 n b}{2 n}\right) S(V, W)-\frac{(a+2 n b) r}{2 n(2 n+1)} g(V, W) \\
& +\left[(a-b)\left\{\frac{r}{2 n(2 n+1)}-\left(\alpha^{2}-\beta^{2}\right)\right\}\right] \eta(V) \eta(W) .
\end{aligned}
$$

From (3.4.3) and (3.4.10), we get 


$$
S(V, W)=2 n\left(\alpha^{2}-\beta^{2}\right) g(V, W)+\left[\frac{b}{a}\left\{r-2 n(2 n+1)\left(\alpha^{2}-\beta^{2}\right)\right\}\right] \eta(V) \eta(Z) .
$$

This implies that the manifold is an $\eta$-Einstein manifold. Hence the theorem is proved. Again, taking $W=\xi$ in (3.4.11) and using (2.13), we get

$$
r=2 n(2 n+1)\left(\alpha^{2}-\beta^{2}\right)
$$

Using (3.4.12) in (3.4.11), we obtain

$$
S(V, W)=2 n\left(\alpha^{2}-\beta^{2}\right) g(V, W) .
$$

This leads to the following:

Theorem 3.5: A trans-Sasakian manifold satisfying the relation $R(X, Y) \cdot \bar{P}=0$ is an Einstein manifold and also is a manifold of constant scalar curvature $2 n(2 n+1)\left(\alpha^{2}-\beta^{2}\right)$.

Now, using (3.4.2), (3.4.3), (3.4.12) and (3.4.13) in (3.4.8), we obtain

which yields

$$
' \bar{P}(U, V, W, Y)=g(\bar{P}(U, V) W, Y)=0,
$$

$$
\bar{P}(U, V) W=0 \text {. }
$$

Therefore the trans-Sasakian manifold under consideration is pseudo-projectively flat. Hence we can state the next theorem:

Theorem 3.6: If in a trans-Sasakian manifold $M$ of dimension $(2 n+1), n>0$, the relation $R(X, Y) \cdot \bar{P}=0$ holds, then the manifold is pseudo-projectively flat.

\section{REFERENCES}

[1] Bagewadi, C S \& Girish Kumar E, Note on Trans-Sasakian Manifolds, Tensor (N.S.), 65(1) (2004) 80.

[2] Bhagawath, P, A pseudo-projective curvature tensor on Riemannian manifold. Bull. of Cal. Math. Soc., 94(3) (2002) 163.

[3] Blair, D E, Contact Manifolds in Riemannian Geometry, Lecture notes in Mathematics, 509, Springer-verlag, Berlin 1976.

[4] Blair, D E \& Oubina, J A, Conformal and related changes of metric on the product of two almost contact manifolds, Publ. Mathematiques, 34 (1990) 199.

[5] De, U C \& Shaikh, A A, Complex Manifold and Contact Manifolds, Narosa Pub. House Pvt. Ltd. (2009).

[6] De, U C \& Tarafdar D, On a type of new tensor in a Riemannian manifold and its relativistic significance, Ranchi Univ. Math. J., 24 (1993) 17.

[7] De, U C \& Tripathi, M M, Ricci tensor in 3-dimensional trans-Sasakian manifol- 
KATHMANDU UNIVERSITY JOURNAL OF SCIENCE, ENGINEERING AND TECHNOLOGY

VOL. 8, No. I, FEBRUARY, 2012, pp 81-87

ds, Kyungpook Math. J., 2 (2003) 247.

[8] Gray, A \& Harvella, L M, The sixteen classes of Almost Hermitian Manifolds and their Linear invariants, Ann. Mat. Pura Appl., 123 (4) (1980) 35.

[9] Oubina, J A, New classes of Almost Contact Metric Structures, Publ. Math. Debre cen, 32, (1985) 187.

[10] Szabo, Z I, Structure Theorems on Riemannian spaces satisfying $R(X, Y) \cdot R=0$, I, the local version, J. Diff. Geo., 17(1980) 531.

[11] Szabo, Z I, Classification and construction of complete hyper-surfaces satisfying $R(X, Y) . R=0$, Acta. Sci. Math., 7 (1984) 321. 